\title{
Social determinants and maternal exposure to intimate partner violence of obstetric patients with severe maternal morbidity in the intensive care unit: a systematic review protocol
}

Beatriz Paulina Ayala Quintanilla, ${ }^{1,2,3}$ Angela Taft, ${ }^{1}$ Susan McDonald, ${ }^{1,2}$ Wendy Pollock, ${ }^{1,2,4}$ Joel Christian Roque Henriquez ${ }^{3}$

To cite: Ayala Quintanilla BP, Taft A, McDonald S, et al. Social determinants and maternal exposure to intimate partner violence of obstetric patients with severe maternal morbidity in the intensive care unit: a systematic review protocol. BMJ Open 2016;6: e013270. doi:10.1136/ bmjopen-2016-013270

- Prepublication history and additional material is available. To view please visit the journal (http://dx.doi.org/ 10.1136/bmjopen-2016013270).

Received 1 July 2016 Revised 2 November 2016 Accepted 7 November 2016

CrossMark

For numbered affiliations see end of article.

Correspondence to Dr Beatriz Paulina Ayala Quintanilla; ayalaquintanilla. b@students.latrobe.edu.au

\section{ABSTRACT}

Introduction: Maternal mortality is a potentially preventable public health issue. Maternal morbidity is increasingly of interest to aid the reduction of maternal mortality. Obstetric patients admitted to the intensive care unit (ICU) are an important part of the global burden of maternal morbidity. Social determinants influence health outcomes of pregnant women. Additionally, intimate partner violence has a great negative impact on women's health and pregnancy outcome. However, little is known about the contextual and social aspects of obstetric patients treated in the ICU. This study aimed to conduct a systematic review of the social determinants and exposure to intimate partner violence of obstetric patients admitted to an ICU.

Methods and analysis: A systematic search will be conducted in MEDLINE, CINAHL, ProQuest, LILACS and SciELO from 2000 to 2016. Studies published in English and Spanish will be identified in relation to data reporting on social determinants of health and/or exposure to intimate partner violence of obstetric women, treated in the ICU during pregnancy, childbirth or within 42 days of the end of pregnancy. Two reviewers will independently screen for study eligibility and data extraction. Risk of bias and assessment of the quality of the included studies will be performed by using the Critical Appraisal Skills Programme (CASP) checklist. Data will be analysed and summarised using a narrative description of the available evidence across studies. This systematic review protocol will be reported according to the Preferred Reporting Items for Systematic Reviews and Meta-Analyses Protocols (PRISMA-P) guidelines.

Ethics and dissemination: Since this systematic review will be based on published studies, ethical approval is not required. Findings will be presented at La Trobe University, in Conferences and Congresses, and published in a peer-reviewed journal.

Trial registration number: CRD42016037492.

\section{Strengths and limitations of this study}

- The comprehensive search strategy of this review will identify a wide range of studies from diverse geographic areas, and include studies published in English and Spanish.

- Measures and reporting of social determinants and exposure to intimate partner violence of critically ill obstetric women affected by severe acute maternal morbidity treated in the intensive care unit (ICU) may be absent in the literature.

- It is likely that there will be some variability (related to clinical and/or methodological diversity) in the studies due to the absence of standardised criteria and/or definition for reporting data on severe acute maternal morbidity in the ICU, making it difficult to compare outcomes across different settings and studies.

- Additionally, it is expected that most included studies might be predominantly observational studies.

\section{INTRODUCTION}

Maternal mortality is a tragic event which has a dramatic negative impact on the remaining family members and motherless children. The Sustainable Development Goal 3.1 targets a challenging global commitment for the reduction of maternal mortality ratio (MMR) to $<70$ per 100000 live births by 2030. ${ }^{1}$ However, 830 maternal deaths occur daily across the world representing a global MMR of 216 per 100000 live births. ${ }^{2}$ Maternal mortality is only a small proportion of the global burden of maternal morbidity and has been compared with the tip of the enormous iceberg where the immense base is formed by maternal morbidity cases ${ }^{3-5}$ including patients affected by severe acute maternal morbidity along with their long-term related complications and disabilities. ${ }^{6-10}$ 
Severe acute maternal morbidity is also known as near miss and both terms are often used interchangeably across studies. ${ }^{11}$ The WHO has developed a tool comprising clinical, management and laboratory-based criteria taking into account organ system dysfunction parameters for defining severe maternal complications. ${ }^{11}{ }^{12}$ However, some investigators consider that the application of these criteria may require the use of advanced laboratory measurements, extensive clinical monitoring and availability of well-trained human resources which could be difficult to perform in lowincome countries, ${ }^{13}$ and even in high-income countries. ${ }^{14}$ Thus, there is a lack of internationally accepted criteria for defining severe acute maternal morbidity worldwide and its definition varies across studies. ${ }^{15-23}$

Regardless, the review of severe acute maternal morbidity has emerged as a potential tool to improve the quality of maternity care. ${ }^{11} 20{ }^{24-26}$ It can be used as a complement to maternal deaths review to allow a more comprehensive assessment for improving maternal health and preventing life-threatening obstetric conditions and fatal maternal events. ${ }^{4} 192728$ Pregnancy, childbirth and the postpartum period can be affected by severe acute maternal morbidity; some women encounter devastating conditions which require specialised critical care in the intensive care unit (ICU). The management of obstetric patients in the ICU involves unique challenges due to the physiological changes of pregnancy, the diverse pregnancy-related disorders and the need to care for two lives. ${ }^{29-32}$ Studies have shown that the incidence of ICU admission varies from $0.04 \%$ to $4.54 \%^{22} 3334$ and the main common causes for ICU admission were hypertensive disorders $(0.09 \%$ of deliveries), obstetric haemorrhage $(0.07 \%)$ and sepsis $(0.02 \%) .^{33}$

Many scholars have argued that ICU admission can be considered as an alternative marker for severe maternal morbidity ${ }^{20} 35-37$ including severe acute maternal morbidity. ${ }^{21} 2238$ In agreement with this, the use of ICU admission was previously proven to have high sensitivity $(86.4 \%)$, specificity $(87.8 \%)$ and positive predictive value (0.85) for identifying severe maternal morbidity. ${ }^{20} \quad 37 \quad 39-41$ This may fail to identify some severe pregnancy-related cases because ICU admission depends on diverse factors including patient's condition, guidelines or criteria established by the healthcare facility, resources available within a hospital such as number of beds or healthcare professionals working in the ICU, among others, which may vary across and within settings and countries. ${ }^{11} 353642$ However, the study of this particular population of obstetric patients provides valuable information since obstetric patients treated in the ICU represent the most critically ill patients and have shown to be an important component of the maternal morbidity spectrum requiring timely managed care. $^{22} 43$ Additionally, the profile of ICU admission has been shown to be similar worldwide, albeit with a higher maternal mortality rate in the ICUs of developing countries. ${ }^{33}$ Thus, it also seems appropriate to use ICU admission to study maternal characteristics and associated factors of obstetric patients with severe maternal morbidity in the ICU. ${ }^{43}$

This wide gap in health outcomes reflects disparities between developed and developing countries resulting from a combination of factors related to social determinants. These include difficulties in accessing healthcare services, gender inequalities, type of healthcare system, poverty level, educational attainment, economic, social and behavioural factors, among others. ${ }^{44-48}$ Social determinants shape the exposure and vulnerability of populations ${ }^{49}$ playing an important role in the health of women and their newborns. ${ }^{50}$ Studies on maternal morbidity should not be limited to looking at medical causes; it is also important to consider the contribution of social determinants and other factors influencing health outcomes at the individual, societal and health system level and their effect on the continuum of care for improving maternal health. ${ }^{547485152}$

Additionally, violence against women is one of the major public health issues and a violation of human rights ${ }^{53} 54$ and many women endure in silence this abuse which is usually exerted by their intimate partners. Globally, $30 \%$ of women have experienced intimate partner violence (IPV) during their lifetime. ${ }^{53} 5556$ However, the prevalence of IPV has a wide range across studies. Garcia-Moreno et a $a \tilde{l}^{7}$ reported that the lifetime prevalence of physical or sexual IPV or both was from $15 \%$ to $71 \%$; and Fulu et a $\bar{l}^{58}$ found in a study where 10178 men were interviewed that the prevalence of physical or sexual violence, or both, was between $25.4 \%$ and $80.0 \%$, and this prevalence was higher after including emotional or economic abuse (39.3$87.3 \%$ ). Furthermore, the rate of IPV during pregnancy was from $1.2 \%$ to $38 \% .{ }^{59}$ Previous systematic reviews on violence in pregnancy have reported that the prevalence of IPV against pregnant women ranged from $0.9 \%$ to $20.1 \%$ in developed countries, ${ }^{60} 2 \%$ to $57 \%$ in African countries $^{61}$ and $3 \%$ to $44 \%$ in Latin America and Caribbean countries. ${ }^{62}$

In addition, there has been increased concern regarding the negative contribution of violence against women to maternal deaths, ${ }^{63-66}$ and many studies have reported negative and fatal repercussions of IPV on women's health ${ }^{6768}$ including during pregnancy and the puerperium period where women could be more vulnerable to partner abuse. ${ }^{62} 64676970$ However, little is known about the underlying factors that undermine maternal health and most studies have no focus particularly on contextual and social aspects including the influence of social determinants and the impact of IPV on critically ill obstetric patients affected by severe maternal morbidity in the ICU.

\section{HYPOTHESIS}

Some social determinants and/or the exposure to IPV influence ICU admission of obstetric patients. 


\section{REVIEW QUESTIONS}

- What are the social determinants described among obstetric patients admitted to an ICU?

- Has exposure to IPV of obstetric patients admitted to an ICU been reported?

- What other characteristics and outcomes are reported among obstetric patients admitted to an ICU?

\section{OBJECTIVES}

- To review available evidence pertaining to social determinants as well as exposure to IPV of obstetric patients admitted to an ICU.

- To review other characteristics and outcomes of obstetric patients admitted to an ICU.

\section{METHODS}

This systematic review protocol will be developed and reported according to the guidelines of Preferred Reporting Items for Systematic Reviews and Meta-Analyses Protocols (PRISMA-P), ${ }^{71} 72$ which include the use of the PRISMA-P checklist (see online supplementary appendix 1 ), and will follow the methodology published previously. ${ }^{33}$ The final review will be also reported considering the recommended items to be addressed in a systematic review in accordance with the PRISMA statement. ${ }^{73}$

\section{Study registration}

This review protocol is registered in the PROSPERO International Prospective Register of Systematic Reviews with registration number CRD42016037492 (http://www. crd.york.ac.uk/PROSPERO/display_record.asp?ID=CRD 42016037492).

\section{Types of studies}

Any studies with relevant data related to social determinants and/or exposure to IPV of obstetric patients admitted to an ICU. These could be experimental and observational studies including randomised controlled trials, nonrandomised controlled trials, quasi-experimental, longitudinal studies, cohort studies, case-control studies and cross-sectional studies.

\section{Types of participants and settings}

Women who were treated in the ICU during pregnancy, childbirth and within 42 days of the end of pregnancy including postpartum, abortion and ectopic pregnancy. This review will consider any healthcare facility which has an ICU, and independent private or public ICUs.

\section{Intervention(s), exposure(s)}

Any social determinants (including age, level of education, marital status, income, place of residence, occupation, socioeconomic status, partner's education, booking status, ethnicity (race), immigration status or country of origin, body mass index, behavioural factors, type of health insurance and others if described) and/or IPV in this study population.

\section{Comparator}

For any identified case-control study, the comparator will be women who were not treated in the ICU during pregnancy, childbirth and within 42 days of the end of pregnancy.

\section{Types of outcome measures \\ Primary outcomes}

The following will be assessed if described in the eligible studies:

- Rate of social determinants identified among obstetric patients admitted to an ICU.

- Rates and types of IPV of obstetric patients admitted to an ICU.

\section{Other outcomes (secondary outcomes)}

The following will be considered if described in the eligible studies:

- Rate of ICU admission (or rate of severe maternal morbidity which includes obstetric death cases).

- Rate of severe acute maternal morbidity (which did not include obstetric death cases) in the ICU.

- Main clinical cause(s) and/or diagnoses for admission to an ICU.

- ICU characteristics including severity of illness, duration of ICU stay and others if indicated (ICU technologies).

- Main obstetric characteristics and/or pre-existing medical conditions of obstetric patients admitted to an ICU.

- Numbers of maternal deaths in the ICU.

- Rate of maternal mortality (case fatality rate, CFR) in the ICU.

- Principal causes of maternal deaths in the ICU.

- Pregnancy and perinatal outcomes.

\section{Search strategy}

A comprehensive systematic literature search will be undertaken between 1 January 2000 and 31 December 2016 in MEDLINE, ProQuest, CINAHL, Latin American and Caribbean Health Science Information Database (LILACS) and SciELO (Scientific Electronic Library Online).

The associations between social inequalities and vulnerabilities, domestic violence and maternal death were first analysed and described in the 1997-1999 UK Confidential Enquiry into Maternal Death, published in December of $2001 .{ }^{74}$ The association of domestic violence and maternal death was recognised by a dedicated chapter for the first time in the 2000-2002 UK Confidential Enquiry into Maternal Death, published in November of 2004. ${ }^{75}$ Earlier and systematic reviews on severe maternal morbidity and ICU admission 2231333576 reported few or no details related to social determinants, and no data on IPV in the maternal ICU admissions. Consequently, this systematic review is targeting the years 2000-2016 to examine if there has been any reporting of social inequalities and vulnerabilities, and IPV in 
studies on maternal admission to an ICU. This time frame is considered appropriate as it parallels the recognition of, and interest in, the association of health determinants and violence against women with maternal mortality, and acknowledges the lack of data reported on these issues in literature reviews covering maternal morbidity studies earlier than 2000 .

We will use the following subject heading and/or freetext words: ICU, intensive care, critical care and critically ill in combination with the next MeSH terms and/or free-text words: social determinants of health, determinants, social, social class, socioeconomic, demographic, characteristics, demographic characteristics, violence, violence against women, gender-based violence, family violence, domestic violence, exposure to violence, battered women, IPV, partner abuse, spouse abuse, spousal abuse, pregnancy, pregnancy complications, pregnancy morbidity, obstetrics, mother, maternity, maternal mortality, maternal death, severe maternal morbidity, severe acute maternal morbidity, near miss, severe obstetric morbidity, partum, childbirth, postpartum, postpartum morbidity, puerperium, parturient and postnatal morbidity.

The general search strategy is shown in online supplementary appendix 2, and will be adapted and modified appropriately according to each database. In addition, hand searching will be also conducted by screening the reference list of eligible articles for further identification of other additional relevant studies. Studies published in English and Spanish will be considered in this review. The first author will conduct the electronic searches and initial identification of studies in MEDLINE, ProQuest, CINAHL, LILACS and SciELO.

It is important to note that there are diverse challenges and barriers during the conduct of a systematic review related to maternal mortality and morbidity. ${ }^{77}$ This is because studies have shown a wide range of criteria to define severe maternal morbidity, severe acute maternal morbidity and maternal admission to an ICU. $^{33} 34$

\section{Data collection and analysis}

\section{Eligibility criteria of the studies}

The inclusion criteria will be:

1. Experimental and observational studies (such as cohort studies, case-control studies, cross-sectional studies).

2. Women admitted to an ICU as stated by the authors in their published research during pregnancy, childbirth or within 42 days of termination of pregnancy (including postpartum, abortion or ectopic pregnancy).

3. The whole (total) population of patients treated in an ICU during pregnancy, childbirth or within 42 days of termination of pregnancy (including postpartum, abortion or ectopic pregnancy).
4. Studies written in English and Spanish between the period 2000 and 2016, which consider as a setting any healthcare facilities with an ICU or independent private or public ICUs.

5. Studies with relevant data related to social determinants and/or IPV.

The exclusion criteria will be:

1. Any qualitative investigations, study protocols, theses, case reports, letters, opinions, editorials, weekly reports, congress abstracts or reviews.

2. Studies which evaluated specific condition(s) or disease(s) treated in the ICU during pregnancy, childbirth or within 42 days of the end of pregnancy (including postpartum, abortion or ectopic pregnancy), for example, restricted to just eclampsia or sepsis.

3. Subgroup of participants treated in the ICU during pregnancy, childbirth or within 42 days of the end of pregnancy (including postpartum, abortion or ectopic pregnancy), for example, anaesthetic complications.

4. Duplicate studies that have used the same study population or data (the most recent or relevant publication will be used for studies published in more than one journal).

5. Studies with absence of data in relation to social determinants and/or IPV.

\section{Data management of the studies}

The bibliographic software program Endnote (V.X7) will be used to manage and store relevant studies. Duplicate references will be found and removed by using this software program. A checklist will be developed based on the eligibility criteria of this review. Online supplementary appendix 3 shows the flow diagram through the main phases of a systematic review. ${ }^{73}$

\section{Data selection of the studies}

The screening of potential studies will be assessed independently by two investigators. They will screen titles and abstracts and/or full texts of all non-duplicate studies resulting from the electronic search, and assess eligibility of potential studies. It might be necessary to obtain and read the full text of the studies from this initial stage of the review-before deciding their exclusion-because of the expected variability of reporting and defining severe acute maternal morbidity and ICU admission in the studies. In addition, some studies may not describe adequately in the abstracts sufficient detail to ensure that the selection criteria were met. The first author will obtain full texts of potential eligible studies. If there are any doubts about whether or not the study (ies) should be included at this stage, this (these) study (ies) will be temporarily included for more evaluation, and proceed to the next stage.

The full version of all selected retained studies will be examined again for further evaluation, taking into account the selection criteria of the studies, by four 
investigators (two for studies written in English, and two for studies written in Spanish).

The final list of selected articles will be reviewed independently. Reasons for exclusion will be documented for each excluded study. Results will be compared. Disagreements will be resolved by discussion and consensus between the two authors. If needed, consultation of a third author will be performed when consensus is not reached. It is expected that the disagreement rate between the two reviewers will not be more than $10 \%{ }^{77}$

\section{Appraisal assessment of methodological quality of the included studies}

It is anticipated that most of the eligible studies will be non-randomised, and there is a wide range of tools for assessing quality and bias of observational studies. ${ }^{78}$ However, evaluating the risk of bias and methodological quality of observational studies might be problematic, and there is no consensus particularly for evaluating risk of bias. $^{79}$ According to Grading of Recommendations, Assessment, Development and Evaluation (GRADE) guidelines, observational studies begin as low-quality evidence which can be rated up. ${ }^{80} 81$ Additionally, the work of the Equator (Enhancing the Quality and Transparency Of health Research) Network (http://www.equator-network. org/about-us/) facilitates transparent and accurate reporting by providing guidelines and tools to allow achieving high standard, reproducibility and usefulness of health studies including study protocols.

For this review, the risk of bias and quality of each included study will be assessed using the Critical Appraisal Skills Programme (CASP) checklist. $^{82}$ This process will be performed independently by four authors (two for studies published in English, and two for studies published in Spanish) and disagreement will be resolved by discussion and consultation of a third author when necessary. Studies will be categorised as very low (unclear), low, moderate or high quality of data.

\section{Data extraction}

Two investigators will independently extract all data items (see online supplementary appendix 4) of each included study by using a standardised data extraction form in accordance with the recommendations of the Cochrane Collaboration and as previously described. ${ }^{33}$ We will ensure that there are no data errors. A third author will randomly cross-check these data. Any disagreements will be resolved by consensus between the two authors, and a third author will act as arbitrator if consensus is not reached.

\section{Data items}

The following descriptive items (see online supplementary appendix 4) will be extracted: (1) general characteristics of the studies, (2) social determinants, (3) IPV characteristics, (4) ICU characteristics, (5) obstetric characteristics, (6) pre-existing medical conditions (comorbidities) and (7) pregnancy and perinatal outcomes.

In summary, we will extract information related to social determinants of health comprising age, marital status, place of residence, socioeconomic status (by using level of education and/or occupation and/or income), partner's education, booking status, ethnicity (race), immigration status or country of origin, body mass index, behavioural factors (smoking, alcohol consumption and use of illicit drugs) and type of health insurance, and others if reported; and exposure to IPV including rates and types of IPV among obstetric patients admitted to an ICU.

Additional information concerning the general characteristics of each study will be extracted: author's name, journal, year of publication, type of design, temporality, setting, country, period and number of participants. Besides, studies will be also examined in relation to ICU characteristics including rate of ICU admission (severe maternal morbidity), rate of severe acute maternal morbidity, main clinical cause(s) and/or diagnoses for ICU admission, length of stay in the ICU, severity of illness, and others if described (ICU technologies); numbers of maternal deaths, maternal mortality rate (CFR) and principal causes of maternal deaths; main obstetric characteristics and pre-existing medical conditions (comorbidities) of the participants; and pregnancy and perinatal outcomes.

The main clinical conditions of severe (acute) maternal morbidity, which were the cause(s) for ICU admission, will include categories previously described: ${ }^{33}$ (1) hypertensive disorders of pregnancy involving (severe) pre-eclampsia, eclampsia and HELLP syndrome; (2) obstetric haemorrhage including antepartum, intrapartum and postpartum causes as reported by individual studies; (3) sepsis/infections; (4) abortions; (5) other direct obstetric complications (included thrombolysis, thromboembolism, pulmonary embolism, pulmonary oedema, acute fatty liver of pregnancy, amniotic fluid embolism, abnormal adherence of placenta, intrauterine fetal death, gestational diabetes and peripartum cardiomyopathy); (6) non-direct obstetric complications which were all other cases not identified as one of the above (including medical conditions and those cases categorised as organ or system failure); and (7) anaesthetic complications.

\section{Data synthesis and analysis}

Data will be synthesised and analysed to answer the research questions. Data will be summarised by country of origin and according to the World Bank's classification of countries by income which consists of four categories: low income, lower middle income, upper middle income and high income. This is based on the gross national income (GNI), per capita of the countries in 2014. Then, low-income countries are those with a GNI per capita $\leq \$ 1045$ in 2014 ; middle-income countries $>\$ 1045$ and $<\$ 12736$; high-income countries 
$\geq \$ 12,736$. Lower middle-income and upper middleincome countries are divided at a GNI per capita of $\$ 4125.83$

Continuous and categorical variables will be summarised according to the presentation of data in each study. It is anticipated that there will be a large variability (related to clinical and methodological diversity ${ }^{84}$ ) of reporting social determinants or exposure to IPV across studies. Then a narrative description of the available evidence will be conducted considering which determinants are significant and their association with the outcome based on data availability of the studies. It will be also indicated if those findings were adjusted for confounders. In a similar manner, data on IPV will be summarised indicating whether or not it was reported, as well as the rate and types of IPV of the eligible studies. Additional data analysis will be made if possible in order to assess the comparisons between studies. ${ }^{33}$

The rate of ICU admission will be mainly shown as the number of obstetric patients per 1000 live births. However, it could be reported as per 1000 deliveries or 1000 maternities or using other denominators according to data shown in each study. These differences are due to the diversity of data reporting ICU admission and lack of consensus in this research area. For those studies which did not report the rate of ICU admission, this value will be calculated if it is possible by using information from the study regarding the number of participants in the ICU per total live births or deliveries or maternities or using other denominators as indicated by the authors.

The rate of the main clinical condition causing ICU admission will be described and/or calculated (if possible) as the number of participants with the specific clinical cause per 1000 deliveries/live births/maternities or using other denominators according to the study. Other ICU characteristics comprising length of stay in the ICU, severity of illness and others if described, as well as principal causes of maternal deaths, numbers of maternal deaths, maternal mortality (case fatality) rate, main obstetric characteristics and pre-existing medical conditions of the participants, pregnancy and perinatal outcomes, will be included by using a narrative summary. Maternal mortality (case fatality) rate will be presented and/or calculated (if possible) as a percentage resulting of the number of maternal deaths per a total number of obstetric patients in the ICU.

This review will present the results as reported in the original studies. However, as indicated previously, we will calculate data, where possible, using the original information from the study such as for rate of ICU admission, rate of main clinical condition causing ICU admission and maternal mortality (case fatality) rate. In addition, data from figures will be used if information is reported either in the text or in the table.

For duplicate studies that have used the same study population or data, the most recent or relevant publication will be used for those studies published in more than one journal, and data might be linked together if needed.

In summary, data analysis will be performed according to data shown in the eligible studies, and statistical expertise will be consulted as needed. The Statistical Package for the Social Sciences (SPSS) V.24 will be used for all the analyses.

\section{Subgroup analyses}

It is planned that subgroup analyses will be performed by considering the World Bank's classification of countries by income (when data are sufficient). In addition, if there is a sufficient number of studies with a design other than cross-sectional, another subgroup analysis will be performed by considering the study design.

\section{Sensitivity analysis}

It is expected that a majority of, or all, studies will be observational (non-randomised) studies. Then a sensitivity analysis will be conducted considering the quality of studies. It will be determined if studies at high risk of bias or only moderate-quality to high-quality studies could change the result of this review if they are included in the study in comparison with when they are not included. Further sensitivity analyses will be considered if necessary.

\section{PRESENTING AND REPORTING THE RESULTS}

The selection process of the studies in the final review will be summarised using a flow diagram according to guidelines of the PRISMA statement (see online supplementary appendix 3) through the main phases of the systematic review consisting of identification, screening, assessment of eligibility and selection of the studies. ${ }^{73}$ Quantitative data of all information for the present systematic review will be shown in tables depending on data shown in the studies by principally indicating the author's name, country and considering the World Bank's classification of countries by income or by study design (when data are sufficient), and accompanied by narrative summaries. The appraisal assessment of quality for each eligible study will be presented in a table as another online supplementary appendix.

\section{POTENTIAL AMENDMENTS}

Amendments to this protocol are not expected. However, if any are required, these amendments will be reported transparently.

\section{CONCLUSION AND IMPLICATIONS}

Severe maternal morbidity is one of the major public health problems which require effective actions to reduce life-threatening obstetric complications leading to ICU admission and maternal deaths. This problem is particularly broad in developing countries where there is a higher MMR since these countries account for $99 \%$ of 
maternal deaths worldwide. However, maternal mortality is potentially preventable; as a consequence, it is important to understand factors influencing severe maternal morbidity, particularly paying attention to social determinants and exposure to IPV of this population of obstetric patients. Social determinants affect outcomes of pregnant women, and IPV has a great negative impact on women's health and during all stages of pregnancy, including an association with maternal mortality. This study will extend knowledge by conducting a systematic review to identify research gaps on severe maternal morbidity, especially in regard to health determinants and IPV.

It is important to note that the findings of this review will be prudently explained and the conclusion will be interpreted cautiously, considering the potential limitations of this study. This is because most included studies will be mainly observational (cross-sectional) studies, which makes it difficult to determine risk factors. Another limitation will be related to the diversity of clinical and methodological approaches used in the included studies and the absence of standardised criteria and/or definition for reporting data related to severe maternal morbidity in the ICU. It might be also possible to lose relevant data since this review includes only studies published in English and Spanish.

Notwithstanding these limitations, this study will provide valuable information and contribute to a better understanding of the global burden of maternal morbidity, and may provide direction and the basis for further studies in obstetric women treated in the ICU particularly affected by severe maternal morbidity.

\section{ETHICS AND DISSEMINATION \\ Ethical issues}

This study will be based on previous published studies and does not involve collection of new or identifiable data. Accordingly, no ethical review is required.

\section{Publication plan}

It is planned that the findings of this review will be presented at La Trobe University, conferences and congresses and form part of the first author's $\mathrm{PhD}$ thesis. The research will be published in a peer-reviewed journal. It is also planned to update this review in future to monitor any changes which may contribute to develop further studies and/or guide health policies.

\section{Registration}

This study has been registered in the International Prospective Register of Systematic Reviews (PROSPERO) with registration number CRD42016037492 (http://www. crd.york.ac.uk/PROSPERO/display_record.asp?ID=CRD 42016037492).

\section{Author affiliations}

${ }^{1}$ The Judith Lumley Centre, La Trobe University, Melbourne, Victoria, Australia
${ }^{2}$ Mercy Hospital for Women, Melbourne, Victoria, Australia

${ }^{3}$ Peruvian National Institute of Health, Lima, Peru

${ }^{4}$ University of Melbourne, Melbourne, Victoria, Australia

Contributors BPAQ, AT, SM and WP conceived and designed the study protocol. BPAQ drafted the manuscript and all authors edited the following versions of the draft. BPAQ, AT, SM, WP and JCRH revised critically the methodological and clinical content of the protocol. All authors have reviewed and approved the final manuscript.

Funding This review is part of a doctoral study at La Trobe University funded by PRONABEC (National Program of Scholarship and Educational Loan), provided by the Peruvian Government, and with the support of La Trobe University postgraduate funding.

Competing interests None declared.

Provenance and peer review Not commissioned; externally peer reviewed.

Open Access This is an Open Access article distributed in accordance with the Creative Commons Attribution Non Commercial (CC BY-NC 4.0) license, which permits others to distribute, remix, adapt, build upon this work noncommercially, and license their derivative works on different terms, provided the original work is properly cited and the use is non-commercial. See: http:// creativecommons.org/licenses/by-nc/4.0/

\section{REFERENCES}

1. United Nations. Sustainable Development Goals. 17 Goals to transform our world. 2015. http://www.un.org/sustainabledevelopment/ (accessed 6 Feb 2016).

2. World Health Organization. Trends in maternal mortality: 1990 to 2015. Estimates by WHO, UNICEF, UNFPA, World Bank Group and the United Nations Population Division. Secondary trends in maternal mortality: 1990 to 2015. Estimates by WHO, UNICEF, UNFPA, World Bank Group and the United Nations Population Division. 2015. http://www.who.int/classifications/icd/factsheet/en/ (accessed 15 Feb 2016).

3. Firoz T, Chou D, von Dadelszen P, et al. Measuring maternal health: focus on maternal morbidity. Bull World Health Organ 2013;91:794-6.

4. Mantel GD, Buchmann E, Rees H, et al. Severe acute maternal morbidity: a pilot study of a definition for a near-miss. Br J Obstet Gynaecol 1998;105:985-90.

5. Geller SE, Cox SM, Callaghan WM, et al. Morbidity and mortality in pregnancy: laying the groundwork for safe motherhood. Womens Health Issues 2006;16:176-88.

6. Ashford L. Hidden suffering: disabilities from pregnancy and childbirth in less developed countries. Secondary hidden suffering: disabilities from pregnancy and childbirth in less developed countries. 2002. http://www.prb.org/pdf/hiddensufferingeng.pdf (accessed 23 Jul 2014).

7. Fottrell E, Kanhonou L, Goufodji S, et al. Risk of psychological distress following severe obstetric complications in Benin: the role of economics, physical health and spousal abuse. Br J Psychiatry 2010;196:18-25.

8. Pacagnella RC, Cecatti JG, Camargo RP, et al. Rationale for a long-term evaluation of the consequences of potentially life-threatening maternal conditions and maternal "near-miss" incidents using a multidimensional approach. J Obstet Gynaecol Can 2010;32:730-8.

9. Wilson RE, Salihu HM. The paradox of obstetric "near misses": converting maternal mortality into morbidity. Int J Fertil Womens Med 2007;52:121-7.

10. Storeng KT, Drabo S, Ganaba R, et al. Mortality after near-miss obstetric complications in Burkina Faso: medical, social and health-care factors. Bull World Health Organ 2012;90:418-25B.

11. Say L, Souza JP, Pattinson RC. Maternal near miss-towards a standard tool for monitoring quality of maternal health care. Best Pract Res Clin Obstet Gynaecol 2009;23:287-96.

12. Haddad SM, Cecatti JG, Souza JP, et al. Applying the materna near miss approach for the evaluation of quality of obstetric care: a worked example from a Multicenter Surveillance Study. Biomed Res Int 2014;2014:989815.

13. Nelissen E, Mduma E, Broerse J, et al. Applicability of the WHO maternal near miss criteria in a low-resource setting. PLOS ONE 2013;8:e61248.

14. Witteveen $\mathrm{T}$, de Koning I, Bezstarosti $\mathrm{H}$, et al. Validating the WHO Maternal Near Miss Tool in a high-income country. Acta Obstet Gynecol Scand 2016;95:106-11. 
15. van Roosmalen J, Zwart J. Severe acute maternal morbidity in high-income countries. Best Pract Res Clin Obstet Gynaecol 2009;23:297-304.

16. Lewis $\mathrm{G}$. Reviewing maternal deaths to make pregnancy safer. Best Pract Res Clin Obstet Gynaecol 2008;22:447-63.

17. Souza JP, Cecatti JG, Parpinelli MA, et al. Appropriate criteria for identification of near-miss maternal morbidity in tertiary care facilities: a cross sectional study. BMC Pregnancy Childbirth 2007;7:20.

18. Donati S, Senatore S, Ronconi A, et al. Obstetric near-miss cases among women admitted to intensive care units in Italy. Acta Obstet Gynecol Scand 2012;91:452-7.

19. David $\mathrm{E}$, Machungo $\mathrm{F}$, Zanconato $\mathrm{G}$, et al. Maternal near miss and maternal deaths in Mozambique: a cross-sectional, region-wide study of 635 consecutive cases assisted in health facilities of Maputo province. BMC Pregnancy Childbirth 2014;14:401.

20. Callaghan WM, Grobman WA, Kilpatrick SJ, et al. Facility-based identification of women with severe maternal morbidity: it is time to start. Obstet Gynecol 2014;123:978-81.

21. Bouvier-Colle $\mathrm{MH}$, Mohangoo AD, Gissler $\mathrm{M}$, et al. What about the mothers? An analysis of maternal mortality and morbidity in perinata health surveillance systems in Europe. BJOG 2012;119:880-9; discussion 90

22. Senanayake $H$, Dias $T$, Jayawardena A. Maternal mortality and morbidity: epidemiology of intensive care admissions in pregnancy. Best Pract Res Clin Obstet Gynaecol 2013;27:811-20.

23. You WB, Chandrasekaran S, Sullivan J, et al. Validation of a scoring system to identify women with near-miss maternal morbidity. Am $J$ Perinatol 2013;30:21-4.

24. Tunçalp Ö, Souza JP. Maternal near-miss audits to improve quality of care. BJOG 2014:121:102-4.

25. Pattinson RC, Hall M. Near misses: a useful adjunct to maternal death enquiries. Br Med Bull 2003;67:231-43.

26. Geller SE, Rosenberg D, Cox SM, et al. Defining a conceptual framework for near-miss maternal morbidity. J Am Med Womens Assoc 2002;57:135-9.

27. Pattinson RC, Buchmann E, Mantel G, et al. Can enquiries into severe acute maternal morbidity act as a surrogate for maternal death enquiries? BJOG 2003;110:889-93.

28. Cecatti JG, Souza JP, Parpinelli MA, et al. Research on severe maternal morbidities and near-misses in Brazil: what we have learned. Reprod Health Matters 2007;15:125-33.

29. Shapiro JM. Critical care of the obstetric patient. J Intensive Care Med 2006;21:278-86.

30. Honiden S, Abdel-Razeq SS, Siegel MD. The management of the critically ill obstetric patient. J Intensive Care Med 2013;28:93-106.

31. Neligan PJ, Laffey JG. Clinical review: special populations-critical illness and pregnancy. Crit Care 2011;15:227.

32. Soubra SH, Guntupalli KK. Critical illness in pregnancy: an overview. Crit Care Med 2005;33:S248-55.

33. Pollock W, Rose L, Dennis CL. Pregnant and postpartum admissions to the intensive care unit: a systematic review. Intensive Care Med 2010;36:1465-74

34. Tunçalp O, Hindin MJ, Souza JP, et al. The prevalence of maternal near miss: a systematic review. BJOG 2012;119:653-61.

35. Gaffney A. Critical care in pregnancy-Is it different? Semin Perinatol 2014:38:329-40.

36. Bouvier-Colle $\mathrm{MH}$, Varnoux N, Salanave B, et al. Case-control study of risk factors for obstetric patients' admission to intensive care units. Eur J Obstet Gynecol Reprod Biol 1997;74:173-7.

37. Kilpatrick SJ, Berg C, Bernstein P, et al. Standardized severe maternal morbidity review: rationale and process. Obstet Gynecol 2014:124:361-6.

38. Reichenheim ME, Zylbersztajn F, Moraes CL, et al. Severe acute obstetric morbidity (near-miss): a review of the relative use of its diagnostic indicators. Arch Gynecol Obstet 2009;280:337-43

39. Geller SE, Rosenberg D, Cox S, et al. A scoring system identified near-miss maternal morbidity during pregnancy. J Clin Epidemiol 2004;57:716-20.

40. Kilpatrick SK, Ecker JL. Severe maternal morbidity: screening and review. Am J Obstet Gynecol 2016;215:B17-22.

41. Main EK, Abreo A, McNulty J, et al. Measuring severe maternal morbidity: validation of potential measures. Am J Obstet Gynecol 2016;214:643.e1-10

42. Brace V, Penney G, Hall M. Quantifying severe maternal morbidity: a Scottish population study. BJOG 2004;111:481-4.

43. Zwart JJ, Dupuis JR, Richters A, et al. Obstetric intensive care unit admission: a 2-year nationwide population-based cohort study. Intensive Care Med 2010;36:256-63.
44. Liljestrand J, Pathmanathan I. Reducing maternal mortality: can we derive policy guidance from developing country experiences? J Public Health Policy 2004;25:299-314.

45. Filippi V, Ronsmans C, Campbell OMR, et al. Maternal health in poor countries: the broader context and a call for action. Lancet 2006;368:1535-41.

46. Echoka E, Makokha A, Dubourg D, et al. Barriers to emergency obstetric care services: accounts of survivors of life threatening obstetric complications in Malindi District, Kenya. Pan Afr Med J 2014;17(Suppl 1):4.

47. Gabrysch S, Campbell OM. Still too far to walk: literature review of the determinants of delivery service use. BMC Pregnancy Childbirth 2009;9:34

48. Say L, Raine R. A systematic review of inequalities in the use of maternal health care in developing countries: examining the scale of the problem and the importance of context. Bull World Health Organ 2007;85:812-19.

49. Solar O, Irwin A. A conceptual framework for action on the social determinants of health. Social determinants of health discussion paper 2 (policy and practice). 2010. http://www.who.int/ sdhconference/resources/ConceptualframeworkforactiononSDH eng.pdf (accessed 18 Sep 2014)

50. United Nation, Every Woman Every Child. EVERY NEWBORN An action plan to end preventable deaths. Secondary EVERY NEWBORN. An action plan to end preventable deaths. 2014 http://www.everynewborn.org/every-newborn-action-plan/ (accessed 14 Mar 2016)

51. Thaddeus S, Maine D. Too far to walk: maternal mortality in context Soc Sci Med 1994;38:1091-110.

52. World Health Organization. Strategies toward ending preventable maternal mortality (EPMM). 2015. http://who.int/reproductivehealth/ topics/maternal_perinatal/epmm/en/ (accessed 28 Mar 2016).

53. World Health Organization. Global and regional estimates of violence against women: prevalence and health effects of intimate partner violence and non-partner sexual violence. 2013. http://apps. who.int/iris/bitstream/10665/85239/1/9789241564625 eng.pdf?ua=1 (accessed 13 Nov 2014).

54. World Conference on Human Rights. Vienna Declaration and Programme of Action. 1993. http://www.ohchr.org/Documents/ Professionallnterest/vienna.pdf (accessed 22 Jul 2014).

55. Devries KM, Mak JY, Garcia-Moreno C, et al. Global health. The global prevalence of intimate partner violence against women. Science 2013;340:1527-8.

56. World Health Organization. The global status report on violence prevention. 2014. http://www.who.int/violence injury prevention/ violence/status_report/2014/en/ (accessed 28 May 2015).

57. Garcia-Moreno C, Jansen HA, Ellsberg M, et al. Prevalence of intimate partner violence: findings from the WHO multi-country study on women's health and domestic violence. Lancet 2006;368:1260-9.

58. Fulu E, Jewkes R, Roselli T, et al. Prevalence of and factors associated with male perpetration of intimate partner violence: findings from the UN Multi-country Cross-sectional Study on Men and Violence in Asia and the Pacific. Lancet Glob Health 2013;1: e187-207.

59. Garcia-Moreno C, Jansen HA, Ellsberg M, et al. WHO Multi-country study on women's health and domestic violence against women. Initial results on prevalence, health outcomes and women's responses. 2005. http://www.who.int/reproductivehealth/publications/ violence/24159358X/en/ (accessed 2 Jul 2014).

60. Gazmararian JA, Lazorick S, Spitz AM, et al. Prevalence of violence against pregnant women. JAMA 1996;275:1915-20.

61. Shamu S, Abrahams N, Temmerman M, et al. A systematic review of African studies on intimate partner violence against pregnant women: prevalence and risk factors. PLOS ONE 2011;6:e17591.

62. Han A, Stewart DE. Maternal and fetal outcomes of intimate partner violence associated with pregnancy in the Latin American and Caribbean region. Int J Gynaecol Obstet 2014;124:6-11.

63. United Nation, Every Woman Every Child. The global strategy for women's, children's and adolescents' health (2016-2030). 2015. http://www.everywomaneverychild.org/global-strategy-2 (accessed 3 Mar 2016)

64. Knight M, Tuffnell DJ, Kenyon S, et al. Saving lives, improving mothers' care-surveillance of maternal deaths in the UK 2011-13 and lessons learned to inform maternity care from the UK and Ireland Confidential Enquiries into Maternal Deaths and Morbidity 2009-13. Oxford: National Perinatal Epidemiology Unit, University of Oxford, 2015. https:// http://www.npeu.ox.ac.uk/ (accessed 15 Apr 2016).

65. Farquhar C, Sadler L, Masson V, et al. Beyond the numbers classifying contributory factors and potentially avoidable maternal 
deaths in New Zealand, 2006-2009. Am J Obstet Gynecol 2011;205:331.e1-8.

66. Knight $\mathrm{M}$, Kenyon $\mathrm{S}$, Brocklehurst $\mathrm{P}$, et al. Saving lives, improving mothers' care-lessons learned to inform future maternity care from the UK and Ireland Confidential Enquiries into Maternal Deaths and Morbidity 2009-12. Oxford: National Perinatal Epidemiology Unit, University of Oxford, 2014. https://www.npeu.ox.ac.uk/downloads/ files/mbrrace-uk/reports/Saving\%20Lives\%20Improving\%20Mothers \%20Care\%20report\%202014\%20Full.pdf (accessed 27 Apr 2016).

67. Black MC. Intimate partner violence and adverse health consequences: implications for clinicians. $J$ Womens Health (Larchmt) 2011;5:428-39.

68. Stöckl H, Devries K, Rotstein A, et al. The global prevalence of intimate partner homicide: a systematic review. Lancet 2013:382:859-65.

69. Alhusen JL, Ray E, Sharps $\mathrm{P}$, et al. Intimate partner violence during pregnancy: maternal and neonatal outcomes. $J$ Womens Health (Larchmt) 2015;24:100-6.

70. Hill A, Pallitto C, McCleary-Sills J, et al. A systematic review and meta-analysis of intimate partner violence during pregnancy and selected birth outcomes. Int J Gynaecol Obstet 2016;133:269-76.

71. Shamseer L, Moher D, Clarke M, et al. Preferred reporting items for systematic review and meta-analysis protocols (PRISMA-P) 2015: elaboration and explanation. BMJ 2015;349:g7647

72. Moher D, Shamseer L, Clarke M, et al. Preferred reporting items for systematic review and meta-analysis protocols (PRISMA-P) 2015 statement. Syst Rev 2015;4:1.

73. Moher D, Liberati A, Tetzlaff J, et al. Preferred reporting items for systematic reviews and meta-analyses: the PRISMA statement. PLoS Med 2009;6:e1000097.

74. The Confidential Enquiries into Maternal Deaths in the United Kingdom. Why mothers die 1997-1999. The fifth report of the Confidential Enquiries into Maternal Deaths. 2001. http://www.hqip.org uk/national-programmes/a-z-of-clinical-outcome-review-programmes/ cmace-reports/ (accessed 2 Aug 2014).

75. Confidential Enquiries into Maternal and Child Health in the United Kingdom. Why mothers die 2000-2002. The sixth report of the
Confidential Enquiries into Maternal Deaths. 2004. http://www.hqip. org.uk/assets/NCAPOP-Library/CMACE-Reports/33.-2004-WhyMothers-Die-2000-2002-The-Sixth-Report-of-the-ConfidentialEnquiries-into-Maternal-Deaths-in-the-UK.pdf (accessed 9 Aug 2014).

76. Baskett TF. Epidemiology of obstetric critical care. Best Pract Res Clin Obstet Gynaecol 2008;22:763-74.

77. Gülmezoglu AM, Say L, Betrán AP, et al. WHO systematic review of maternal mortality and morbidity: methodological issues and challenges. BMC Med Res Methodol 2004;4:16.

78. Sanderson S, Tatt ID, Higgins JP. Tools for assessing quality and susceptibility to bias in observational studies in epidemiology: a systematic review and annotated bibliography. Int $J$ Epidemiol 2007;36:666-76.

79. Higgins JP, Ramsay C, Reeves BC, et al. Issues relating to study design and risk of bias when including non-randomized studies in systematic reviews on the effects of interventions. Res Synth Methods 2013;4:12-25.

80. Guyatt GH, Oxman AD, Sultan S, et al. GRADE guidelines: 9. Rating up the quality of evidence. J Clin Epidemiol 2011;64:1311-16.

81. Balshem $\mathrm{H}$, Helfand M, Schünemann $\mathrm{HJ}$, et al. GRADE guidelines: 3. Rating the quality of evidence. J Clin Epidemiol 2011;64:401-6.

82. Critical Appraisal Skills Programme (CASP). Secondary. http://www. casp-uk.net/ (accessed 24 Oct 2015).

83. World Bank. Data. Country and lending groups. Secondary Data. Country and lending groups. 2016. http://data.worldbank.org/about/ country-and-lending-groups (accessed 4 Nov 2015).

84. Ryan R, Cochrane Consumers and Communication Review Group. Heterogeneity and subgroup analyses in Cochrane Consumers and Communication Review Group reviews: planning the analysis at protocol stage. Secondary heterogeneity and subgroup analyses in Cochrane Consumers and Communication Review Group reviews: planning the analysis at protocol stage. 2014. http://cccrg.cochrane. org/sites/cccrg.cochrane.org/files/uploads/Heterogeneity_subgroup_ analyses.pdf (accessed 1 Dec 2015). 